\title{
Automatic Inspection of Surface Mount Solder Joints Using X-Ray Images
}

\author{
E. Griffiths \& R. Jordan \\ IBM UK Scientific Centre \\ St. Clement's St. \\ Winchester SO23 9DR
}

\begin{abstract}
Surface Mount Technology (used in mamufacture of the IBM PS/2) allows great flexibility in layout and very rapid assembly of densely populated circuit boards. The technique presents problems however in the positioning, soldering and testing of chips with very small gaps between leads $(10$ thousandths of an inch). Anticipated future improvements in the technology will make conventional visual and electrical circuit board testing techniques infeasible as lead pitch decreases and numbers of leads per chip and numbers of chips per board increase. This paper describes an alternative method of detecting defective solder joints by analysing $X$-ray images of the chips using computer vision techniques.
\end{abstract}

This paper describes a project to inspect the solder joints on large Surface Mount Technology (SMT) chips which are being used increasingly in the production of the IBM PS/2. These chips, known as Quad Filat Packs (QFP's), typically have up to 36 leads on each of their four sides with a lead pitch of 25 thousandths of an inch (lead is 15 thousandths wide with a gap of 10 thousandths). The project is a collaboration between manufacturing, development and research departments within IB $\mathrm{M}^{1}$ and was started because Manufacturing anticipate problems with placing and testing QI'Ps reliably.

Currently solder joints are inspected visually with a microscope or tested electrically. Neither is completely satisfactory. Visual inspection is monotonous and faults are difficult to detect using visible light. Electrical tests may indicate a connection between lead and board even when no solder is present on the joint (this may result in intermittent faults during normal use). Probe holes required for electrical testing occupy space around the chip reducing the benefit of component miniaturisation. 'These techniques will be unreliable or impossible in the near future when chips will have even more leads, spaced even more closely.

These methods also have the drawback that they do not provide any qualitative information about why joints failed. For this reason, manufacturing chose to investigate X-ray inspection methods. X-ray images give a good indication of solder joint quality because the solder contains lead which is opaque to $\mathrm{X}$-rays. Early trials showed that the quality of images might be sufficient to allow automation of inspection via computer vision systems.

Although the X-ray/computer vision system has the potential to provide good diagnostic and qualitative information it must be fast; visual inspection of the whole board takes about 6-7 minutes and electrical testing of selected components takes about 30 seconds. Time for inspection by the computer vision system must be of this order. The initial target for processing of one board (i.e. loading/unloading the board, moving the robot and inspecting all QIP's) is 3-5 minutes. This allows less than one second to inspect each image containing $15-20$ joints. The program must also be extremely accurate because Manufacturing make enormous numbers of PS/2s (about 1.5 million/yr) and even small numbers of inaccurate diagnoses could mean unacceptable numbers of faulty $\mathrm{PS} / 2 \mathrm{~s}$ leaving the factory and/or unnecessary manual reinspection of boards. These constraints suggest that the inspection system should make use of prior knowledge about the image (such as location and dimensions of joints) so that code can be made robust, fast and reliable.

\section{DESIGN PRINCIPLES}

\section{Image quality and fault resolution.}

The number of joints fitted into each image should be maximised to reduce the overhead of image capture and transfer but this must be balanced against the minimum resolution required to detect defective joints. Capturing just four images of the four corners of the chip (see Figure 1, top) would make efficient use of the resolution of the frame grabber in each axis while minimising the number of images. In practice this was not possible because the poor image quality of the intensifier/video camera combination meant that resolution would be too low to detect faults at this magnification. Images can be corrected for geometric and brightness distortion but still appear fuzry due to inherent limitations in the machinc's optical system. The number of joints that could be inspected in one image was about 20 with a resolution of about 20 pixels across each joint (sec ligure 1, bottom), requiring 8 images per chip but allowing placement of the joints in the least distorted central region of the frame.

1 The IBM Manufacturing Plant in Grecnock, the UK Devclopment Manufacturing Process Centre in Hursley, and the UK Scientific Centre in Winchester. 

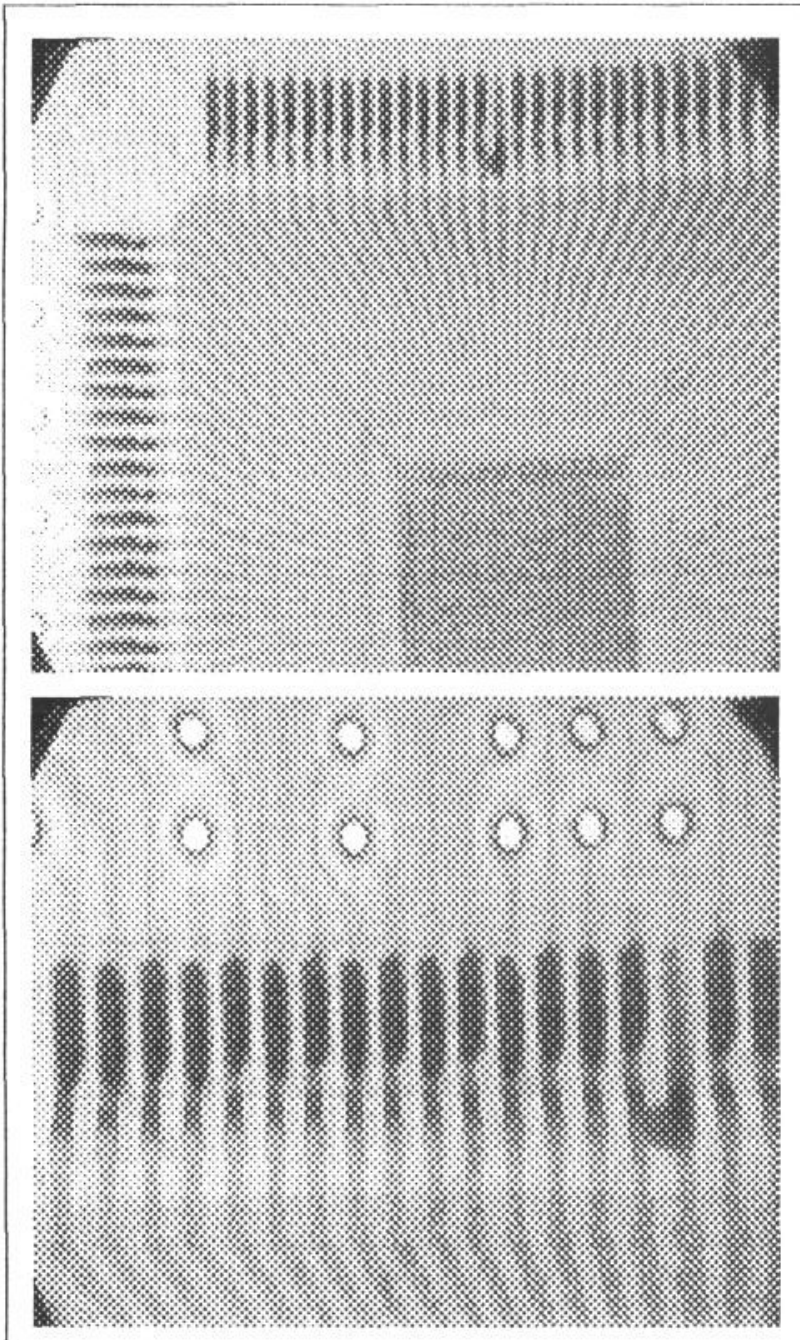

Figure 1. Video X-ray image of one corner and half of one side of a QFP.

\section{Illumination and solder detection.}

The inspection technique depends on the premise that there is a direct and consistent relationship between pixel brightness in the image and solder thickness on the board. It follows that careful control of image illumination is vital. For instance, the Automatic Gain Control provided by the video camcra had to be disabled to ensure that pixels within joints are not affected by other dark or light objects which happen to fall within the image. The image is also subject to gradual and sudden illumination changes. Gradual changes (which may be caused by the video camera warming up over time) can be overcome by adjusting image aquistion parameters each time a new board is inspected. Sudden changes (such as the X-ray filament burning out) can be deected by a rapid check of the contrast of each image.

\section{Joint location.}

Significant savings in runtime and complexity of programs can be achieved and robustness increased if the position of the joints in the image can be carefully controlled:

- The search space in which to locate the joints is greatly reduced.

- Lrrors in robot movement, placement of the card in the robot gripper or placement of the original chip can be detected because joints will appear in the wrong place in the image. Given the scheme (outlined above) of inspecting half a side of a chip in each image, accurate robot movement is essential to ensure that joints are not ignored.

- The number of joints in each image is predetermined so errors in finding individual joints will be detected.

- The resolution per joint is fixed allowing more robust code which has a 'scale to work to'.

\section{Fault identification.}

It's tempting to collect a sample of images which show bad joints and write an algorithm to detect each of the possible faults. An alternative is to collect a number of carefully selected measurements from cach joint, which characterise joint quality in a very compact form. These measurements can be analysed by standard classification algorithms $\mid 1,2$, $3 /$ in order to identify possible defects. The latter approach has the following advantages:

- Manufacturing processes change frequently, introducing new faults. If the "fault-finding" approach was used, new algorithms would have to be coded for each new fault. With the classification approach, it is only necessary to re-train the classifier.

- A multitude of fault-finding algorithms will require many tuncable parameters. Parameters are complex to tune, and there is no guarantee that the best settings will ever be found. Tuning a classificr is automatically done as part of the training process.

- It is not always vital to report the precise nature of the fault, since defective joints will be reworked manually, at which point the defect will become obvious. $\Lambda$ classifier may highlight a joint which is significantly different from normal, even if it does not fit any of our preconceived categories of defect.

- The classifier may be more efficient for two reasons; there is bound to be redundancy involved in cxccuting a scrics of fault finding tests, all of which must be passed before a joint is judged to be good, there may be more faults to be tested for than there are joint quality measurements to be made. 


\section{THE SYSTEM}

\section{Hardware.}

The equipment used is shown in Figure 2. The $\mathrm{X}$-rays are projected in a 40 degree cone from a fine filament through the inspection target and picked up by an intensifier tube which converts the X-ray photons into visible light which is then picked up by a standard video camera. The images are then grabbed by the workstation for analysis. The most important feature of the system is its ability to zoom in (by moving the target closer to the X-ray source) and provide any resolution that might be required for the inspection of joints in the foreseeable future.

\section{Software.}

The automatic solder joint inspection program performs the following tasks:

- Reads a configuration file containing characteristics of the board to be inspected and options for the run

- Records and displays debugging information if required

- Signals the robot to move from chip to chip on the circuit board

- Captures X-ray images of the large SMT chips

- Corrects the images for geometric and brightness distortion

- Finds the window containing the row of joints in the image

- Checks the image quality of the joint window

- Finds individual joints within the window

- Makes measurements of joint quality

- Assesses joints using classification algorithms.

- Compiles an inspection report listing joints which are defective and makes suggestions as to why they failed

Image distortion: Images were distorted in their geometry and brightness and showed random noise. Averaging over up to 10 frames produced a small reduction in noisc but not sufficient to enhance fault assessment so no averaging was done. Both the uneven brightness and geometric distortion are the result of projecting the cone-shaped X-ray beam onto a flat plane. This is compounded by the cone centre not usually falling in the centre of the image. Both of these factors will change gradually as the filament wears out and drastically when the filament is replaced producing a different alignment. Correction of these two factors is a two stage process. Distortion is measured using a known target and modelled using polynomials. The polynomials are evaluated at each pixel in the imagc, and placed in a look up table. Construction of the look up table is time-consuming, and is carried out during an infrequent calibration process. During an inspection run individual images can be corrected very rapidly by looking up a correction factor rather than by recalculation.

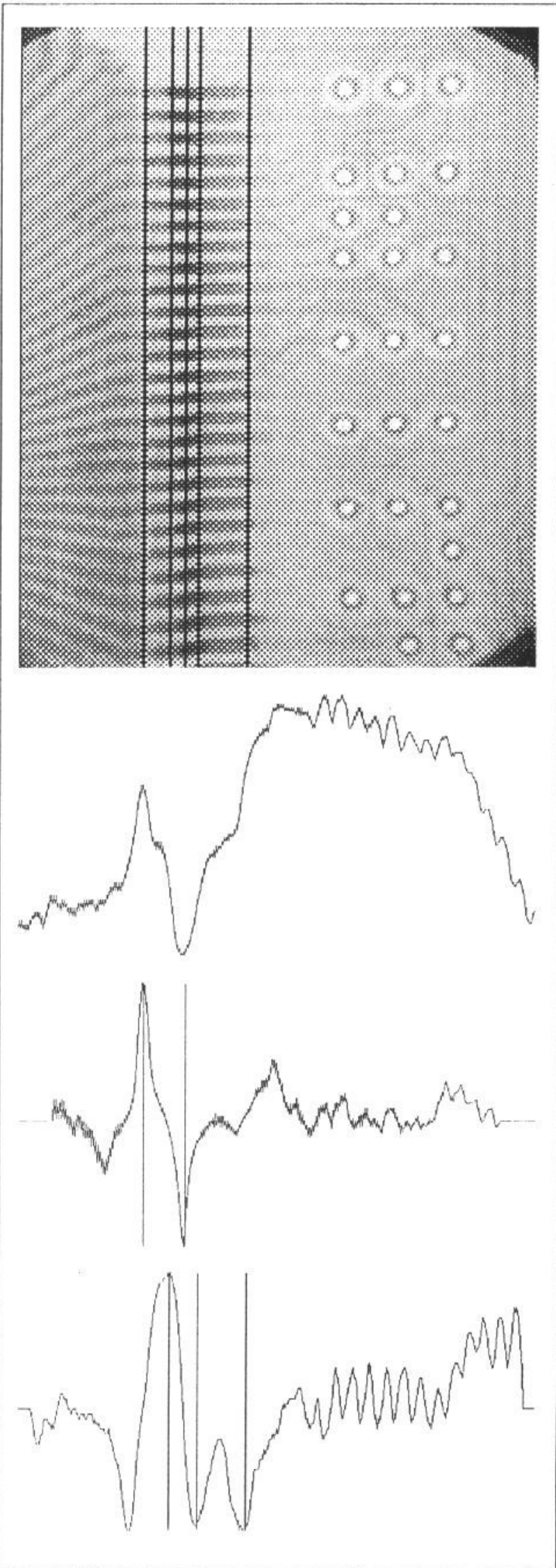

Iigure 3. X-ray image showing typical cross-section of row of joints, with curvature and Ist difference cdge maps of the profile.

I ocating the joint window: Location of the row of joints in the image needed to be independent of 


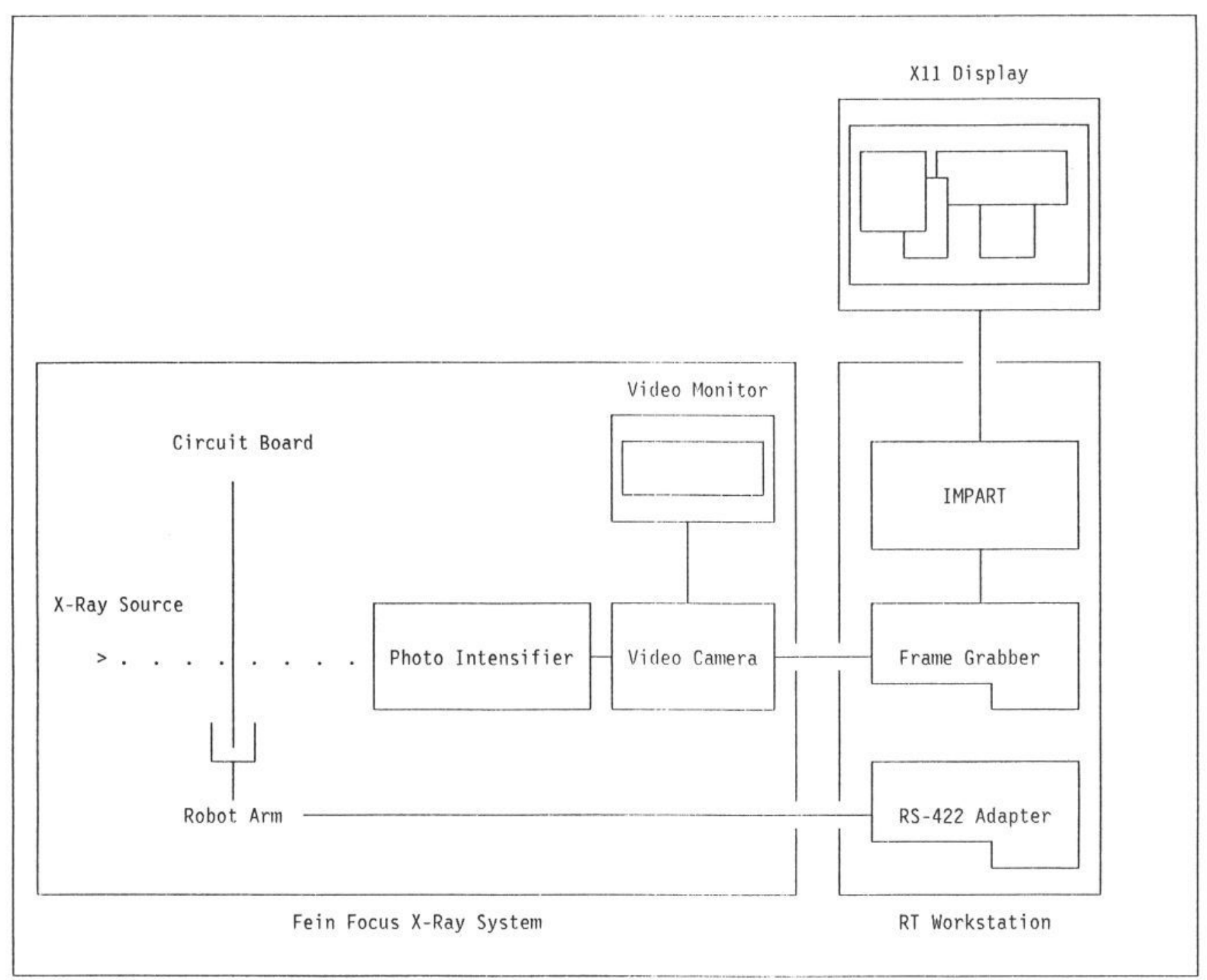

Figure 2. Equipment Used for X-Ray Image Generation and Capture

- Irrelevant features close to the joint row

- Changes in overall brightness/contrast

- Errors in position of the joint row (due to chip placement or robot movement)

- The ratio of good and bad joints in the row

- Slight skewing of the joint row due to bad placement

Doing a projection of the image along the row of joints produces a characteristic profile showing the chip body, the lead between chip and joint and the joint itself (see Figure 3 ). The profile shows charateristic peaks, troughs and edges which identify the position of the joint and surrounding landmarks such as the leads between joint and chip body and the lead knees (all leads have a 'knce' formed where they bend down from the chip to the board). Using prior knowledge about the distribution of these features to analyse the shape of the profile produced an algorithm that was robust to the problems listed above. The peak representing the lead between joint and chip body and the trough representing the lead knee were found by measuring curvature (calculated from first and second differences $[4,5]$ ) along the profile at the scale of the peak and trough (see Figure 3). The positions of these features are used as the starting point to search for the significant edges
(Ist difference) in the profile which identify the top and bottom of the joints.

Once these features have been located two subimages are extracted, one containing the knees and one containing the joints. The subimages are transposed and/or mirrored such that all windows have the same orientation. This makes subsequent code simpler and faster by avoiding a decision tree based on the orientation of the image (which may be horizontal or vertical, top or bottom, left or right).

Checking window image quality: The joint window is found even if small faults in the imaging system have altered the brightness/contrast of the image. Quality of the image must now be checked to ensure that these errors will not affect solder measurements. The problem is to find some part of the image which presents consistent grey scale differences. Joints cannot be used as they have variable amounts of solder. Fortunately the knec which is present on all leads presents a sufficient thickness of metal to make it very dark in the image while the spaces in between knees are board only and hence very bright in the image. The knecs provide an ideal 'black, white, black....' strip which can be used to check contrast and brightness. Overall brightness and contrast of the knee window is checked and the brightness of 
the knees versus background is also checked to make sure they do not overlap as this would signal that solder measurements may be inaccurate.

Checking joint placement in the window: Precise placement of the joints in the image is vital if joints are not to be missed. This is checked by looking for the knee at the end of the joint row in each image, which should be neither too close nor too far away from the end of the image. Joints could not be used in this test because of instances when the whole chip has been misplaced during mounting by one lead. In this case, the last joint has no solder and is difficult to detect. The lead can still be detected by the position of its knee and the misplacement reported.

Finding the joints within the window: Edges of chip leads (including those without solder) are found in the joint window by convolution with a first difference edge-detector. $\Lambda$ s joints are always aligned horizontally or vertically in the image, significant savings in the convolution process can be made by first projecting across the joint row to give a grey-level profile of the joints (see Figure 4) and then convolving this vector with a 1 -dimensional edge detector $(-1,-1,0,1,1)$. This technique has three major advantages: greatly increased speed by reduction of the amount of data to be convolved, reduced noise in the final edge profile because projection averages the grey-level along the joints, and the convolution itself is reduced to a 1-I) mask and image. The final result of the

projection/convolution is an edge strength vector across the chip leads (see Figure 4).

The edge vector may show peaks resulting from noise or voids in joints. The real joint edges are distinguished by a process of smoothing, thresholding and thinning:

- Smoothing: The raw edge vector may be smoothed by local averaging. Smoothing can remove small sub-maximal edge strengths. But in some cases (e.g. main edge strength has small, adjacent, sub maximal edge strength) this has the effect of moving the observed maximal edge strength by a few pixcls. For this reason no smoothing is used in the current inspection scheme.

- Thresholding: Only those edge points with an edge strength exceeding some user-specified threshold arc accepted for later processing. This value has been tuned by trial and error. It is acceptable for a few spurious edges to remain after thresholding, but no real edges should be lost by this process.

- Thinning: The even spacing of the joints is exploited to eliminate a few spurious edges which remain after thresholding. If the proportion of spurious edge points remaining after thresholding is high (approaching 50 per cent), this process can do more harm than good, and so it is not attempted.

The thresholded edge points are compiled into two lists containing the coordinates of positive and negative edge points. The median distance between adjacent joints is calculated for each list. Provided there is good agreement between the positive median, the negative median and the expected lead pitch, this empirically discovered median is

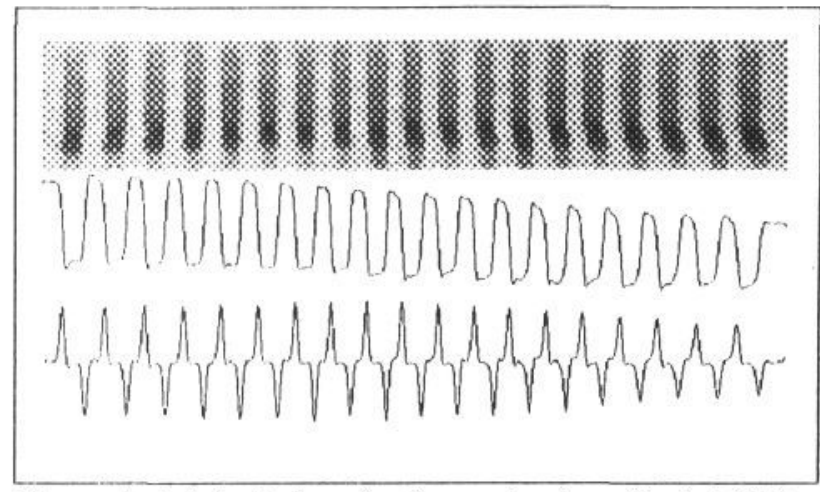

Figure 4. Joint window (top), grey-level profile (middle) and edge vector (bottom).

accepted as a measure of lead pitch in pixels. Each point on each list is examined to see whether the inter-edge distance between its two neighbours is closer to the lead pitch than the distance between the point and its neighbour. If this is the case, the point is removed from the list. (See Figure 5).

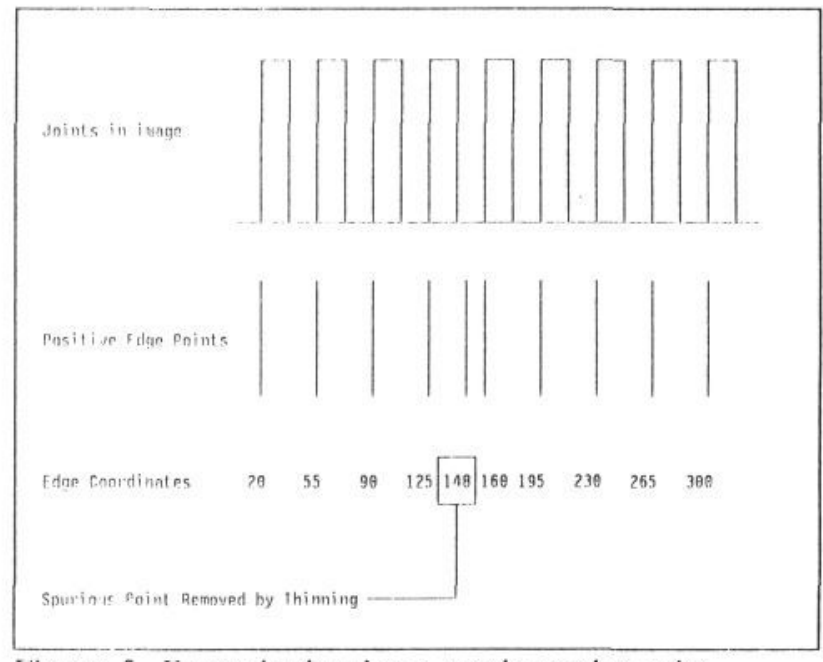

Figure 5. Eixample showing a spurious edge point removed by thinning.

After identifying joint edges, we are left with a list of positive edge points and a list of negative edge points. $\Lambda$ joint is represented by the coordinates of its two edges, so the two lists must be merged to form the joint list. This involves taking each edge from the positive edge list and pairing it with a point from the negative edge list. The point from the negative list is chosen such that the resulting joint width approximates as closely as possible to the known lcad width. Implausible results such as overlapping joints, missing or spurious edges are checked and avoided or reported if not recoverable. These positions are then back-projected into the joint window for the examination of solder distribution along the joint.

Measuring joint quality: The joint window defines an area containing joints (delimited by joint edges) and the spaces between joints. The distribution of solder within these areas is assessed by measurement of mean pixcl values. Sampling of these areas could be exhaustive, but three factors make this unnecessary. lirstly, the high surface tension of molten solder means that there is a minimum area that solder can cover. In practice this means that only the 
edges of the joints need be searched. Secondly, a glance at the cross-sectional shape of the joint (Figure 6) shows that the search region must be offset by a few pixels from the maximal edge if the grey level comparison is to reflect the true difference between joint and background. Thirdly the space between joints only needs to be searched down the middle to establish whether solder from either joint has flowed into the space in between. Each search region is further subdivided into four, to improve detection of uneven solder distribution, which may be caused by bridges or partially soldered joints. The actual search regions are shown in Figure 6. Measurement of joint quality also includes maximum edge strength, overall solder distribution and similarity to neighbouring joints.

Classification of joints: Bayesian classification algorithms are used to distinguish good and defective joints, based on the measurements of joint quality described above. Details of the classification techniques are beyond the scope of this paper.

\section{RESULTS}

Eleven circuit boards each containing fourteen QIPS giving a total of 17,424 joints were manually examined to determine the true frequency of solder joint faults. The boards were then inspected using our program. Results from these trials look promising ('Table 1) as the frequency of misdiagnosed faults is low. It is misleading, however, to concentrate on these overall figures because the frequency of faults is itself very low $(0.2 \%)$. $\Lambda$ more realistic analysis would be that 2 out of $34(6 \%)$ of actual faults were missed, i.e. diagnosed falsely as good joints, and 20) out of $52(38 \%)$ faults diagnosed by the system were actually good joints. The implications of these results for manufacturing are discussed briefly below.

\begin{tabular}{|l|l|l|l|l|}
\hline \multicolumn{4}{|c|}{ Table 1. Solder joint inspection results. } \\
\hline & TRUE & \multicolumn{2}{|l|}{ FAISE } \\
\hline & Good & Bad & Good & Bad \\
\hline Number & 17370 & 32 & 2 & 20 \\
\hline Percent & 99.69 & 0.18 & 0.01 & 0.12 \\
\hline
\end{tabular}

\section{DISCUSSION}

We have described a method of automatically inspecting the solder joints of SMT components using X-ray images. Our experience indicates that the success of such a project hinges on a balance of design principles: choice of an appropriate scale at which to work, careful control of image illumination and component placement within the image, and a

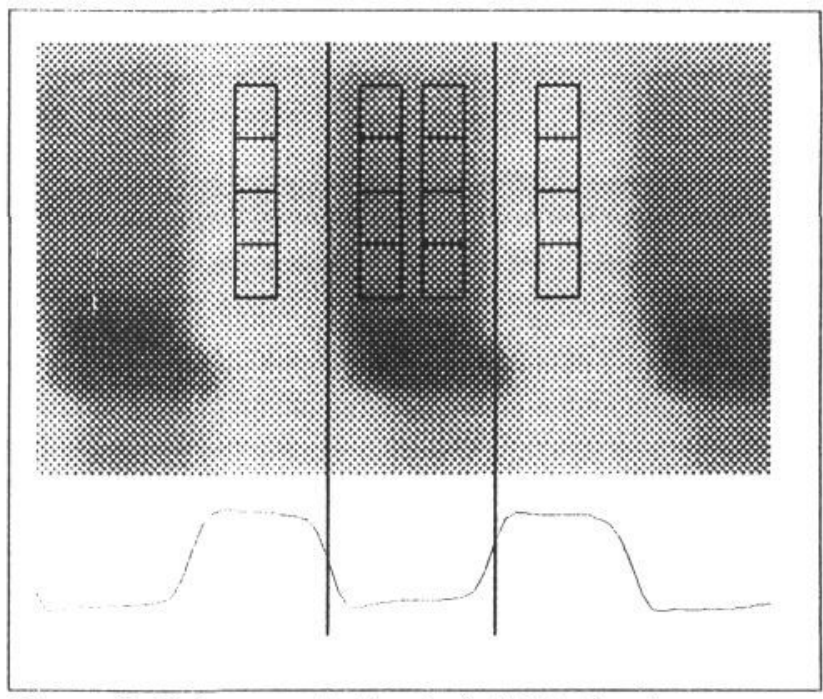

ligure 6. Enlargement of a single joint, showing crossscctional shape and solder measurement regions.

gencralised approach to fault detection and of course system performance. Our algorithms enable us to reliably locate joints within an image and then take a series of measurements which are input to a Bayesian classifier to characterise joints as 'good' or 'bad'.

Work in progress will allow us to determine whether additional measurements can improve the accuracy with which joints are diagnosed. The current rates of false diagnoses are unlikcly to be acceptable to Manufacturing for full inspection of every board produced: every bad joint diagnosed as good represents a faulty $\mathrm{P}$ 'S $/ 2$ going to a customer, every good joint diagnosed as bad represents lost revenue to IB $\mathrm{M}$ in terms of the board being returned unnecessarily for rework. The cnormous volumes of boards produced by Manufacturing mean that software diagnosis will have to be close to perfect.

\section{REFERENCES}

1. James, M. Classification Algorithms. Collins, UK. (1985).

2. Iukunaga, $\boldsymbol{K}$. Introduction to Statistical Pattern Recognition. Academic Press (1972).

3. Lippmann, R.P. "Pattern Classification Using Neural Networks" IEEE Communications Magazinc (USA), Vol. 27 (1989) pp. 47-50, 59-64.

4. Iaux, II.\& Pratt, M.J. Computational Geometry for Design and Manufacture Ellis IIorwood Ltd., UK. (1981).

5. Smith, G.D. Numerical Solution of Partial Differential Equations: Finite difference Methods Clarendon Press, UK. (1985). 